Classification

Physics Abstracts

$75.55-71.70-75.30 \mathrm{H}-75.30 \mathrm{E}$

\title{
Acceptors in wide-gap semimagnetic semiconductors
}

\author{
J. Mycielski
}

Groupe de Physique des Solides de l'Ecole Normale Supérieure, 24 rue Lhomond, 75231 Paris Cedex 05, France and Institute of Theoretical Physics, Warsaw University, 00-681 Warsaw, Poland (*).

and C. Rigaux

Groupe de Physique des Solides de l'Ecole Normale Supérieure, 24 rue Lhomond, 75231 Paris Cedex 05, France

(Reçu le 10 mars 1983, accepté le 30 mai 1983)

\begin{abstract}
Résumé. - Nous considérons les états accepteurs peu profonds dans les semiconducteurs semimagnétiques de large bande interdite, appartenant au réseau blende de zinc (comme $\mathrm{Cd}_{1-x} \mathbf{M n}_{x} \mathrm{Te}$ ). Le quadruplet fondamental de l'accepteur est déterminé par la méthode variationnelle de Schechter. Du fait de l'interaction d'échange avec les ions magnétiques, la dégénérescence du quadruplet fondamental est levée. La séparation énergétique des composantes du quadruplet est plus faible que celle des niveaux de trous libres. Les éléments de matrice des transitions entre les niveaux accepteurs et les niveaux de Landau de la bande de conduction sont également calculés.
\end{abstract}

\begin{abstract}
Shallow acceptors in wide-gap zinc-blende-type semimagnetic semiconductors (like $\mathrm{Cd}_{1-x} \mathbf{M n}_{\mathbf{x}} \mathrm{Te}$ ) are considered. The four-fold degenerate acceptor ground state is determined using Schechter's variational method. The exchange interaction with magnetic ions is then included and the splitting of the ground state is studied. It is shown that this splitting is quenched as compared with the free-hole splitting. Matrix elements for the acceptor to conduction Landau states transitions are also calculated.
\end{abstract}

\section{Introduction.}

The so-called "semimagnetic semiconductors" (or "diluted magnetic semiconductors") are alloys of non-magnetic and magnetic semiconductors (see the review articles 1-8). In the present paper we will be interested in wide-gap semimagnetic semiconductors of the zinc-blende cubic crystal structure. Probably the best known material of this sort is $\mathrm{Cd}_{1-x} \mathrm{Mn}_{x} \mathrm{Te}$.

The specific features of the semimagnetic semiconductors follow from the exchange interaction of the magnetic ions with conduction 'and valence band electrons. This interaction strongly affects the band structure [9-11] and the impurity states [5, 8]. In particular, the top of the valence band - the four-fold degenerate $\Gamma_{8}$ level - is split by the exchange interaction.

In narrow-gap semimagnetic semiconductors, like $\mathrm{Hg}_{1-x} \mathrm{Mn}_{x} \mathrm{Te}(x>0.075)$, both the Landau and the

(*) Permanent address. exchange splittings are larger than the shallow acceptor binding energy already at few Tesla external magnetic field, due to the low effective masses of heavy and light holes. The acceptor states are then drastically changed and, in particular, the acceptor binding energy is lowered (" boil-off » effect) [12-15].

In wide-gap semimagnetic semiconductors the Landau splitting is smaller and the shallow acceptor binding energy is higher due to high effective masses of holes. Therefore, the effect of the magnetic field and the exchange interaction on the acceptor states is weaker, resulting mainly in splitting. This was indirectly observed in $\mathrm{p}-\mathrm{Cd}_{1-x} \mathrm{Mn}_{x} \mathrm{Te}$ [16]. The purpose of the present paper is to calculate the acceptor ground state splitting in a relatively simple way. We start with determining the acceptor's ground-state wave functions in the absence of exchange interaction, using Schechter's variational method [17]. We present this method in an explicit and simplified way. Then we include the exchange interaction and calculate the ground-state splitting. It follows that this splitting is smaller than the splitting of the free-hole $\Gamma_{8}$ level. 
We also calculate the matrix elements for the acceptor to conduction Landau states transitions.

The effect of the magnetic field and the exchange interaction on the acceptor states can be calculated in a more general way, for both narrow- and wide-gap semimagnetic semiconductors and in the whole range of magnetic fields [18], using the spherical tensor operators and a reduced matrix elements technique $[19,20]$. However, a much more elaborate variational procedure has to be used and the results are less explicitly dependent on the material parameters.

It should be noted that there exists a feedback : the localized spins of the magnetic ions not only influence through the exchange interaction the hole on the acceptor but this hole may also produce some ordering of the surrounding localized spins (see the review article 21). The latter effect - formation of the bound magnetic polaron - will not be discussed in the present paper.

\section{Acceptor in the absence of exchange.}

In the following we will be interested only in widegap zinc-blende-structure semiconductors with large spin-orbit splitting of the valence band (e.g., $\left.\mathrm{Cd}_{1-x} \mathrm{Mn}_{x} \mathrm{Te}\right)$. The four-fold degenerate electron top state of the valence band transforms according to the $\Gamma_{8}$ representation of the double $T_{\mathrm{d}}$ point group. The acceptor wave functions can be constructed from the orthonormal basis of this $\Gamma_{8}$ level, which is (we use the reference system of cubic crystallographic axes)

$\Phi_{1}=\left|\frac{3}{2}, \frac{3}{2}\right\rangle=2^{-1 / 2}|(X+i Y) \uparrow\rangle$,

$\Phi_{2}=\left|\frac{3}{2}, \frac{1}{2}\right\rangle=i 6^{-1 / 2}|(X+i Y) \downarrow-2 Z \uparrow\rangle$,

$\Phi_{3}=\left|\frac{3}{2},-\frac{1}{2}\right\rangle=6^{-1 / 2}|(X-i Y) \uparrow+2 Z \downarrow\rangle$,

$\Phi_{4}=\left|\frac{3}{2},-\frac{3}{2}\right\rangle=i 2^{-1 / 2}|(X-i Y) \downarrow\rangle$.

The position-dependent functions $X, Y$ and $Z$ transform under the operations of $T_{\mathrm{d}}$ point group as p-type functions, and have average square modulus equal to 1 .

In the absence of magnetic field and exchange interaction the wave functions of the four-fold degenerate ground state of a shallow acceptor have the form

$$
\psi_{i}=\sum_{j=1}^{4} \Phi_{j} \varphi_{j i}, \quad i=1, \ldots, 4,
$$

where $\varphi_{j i}$ are slowly-varying envelope functions of the position $\underline{r}$. The orthonormalization of the functions $\psi_{i}$ yields the condition

$$
\sum_{j=1}^{4}\left\langle\varphi_{j i} \mid \varphi_{j i^{\prime}}\right\rangle=\delta_{i i^{\prime}}
$$

We use the Schechter's variational method for determining the acceptor ground state (for infinite spin-orbit splitting) [17]. Thus, we take the $\varphi_{j i}$ functions in the form $[17,22]$

$$
\begin{aligned}
\left(\begin{array}{l}
\varphi_{11} \\
\varphi_{21} \\
\varphi_{31} \\
\varphi_{41}
\end{array}\right) & =\left(\begin{array}{c}
c_{1} \exp \left(-r / r_{1}\right)+\frac{1}{2} c_{2}\left(3 z^{2}-r^{2}\right) \exp \left(-r / r_{2}\right) \\
i c_{3} z(x+i y) \exp \left(-r / r_{2}\right) \\
{\left[-\left(3^{1 / 2} / 2\right) c_{2}\left(x^{2}-y^{2}\right)+i c_{3} x y\right] \exp \left(-r / r_{2}\right)} \\
0
\end{array}\right) \\
\left(\begin{array}{l}
\varphi_{12} \\
\varphi_{22} \\
\varphi_{32} \\
\varphi_{42}
\end{array}\right) & =\left(\begin{array}{c}
-i c_{3} z(x-i y) \exp \left(-r / r_{2}\right) \\
c_{1} \exp \left(-r / r_{1}\right)-\frac{1}{2} c_{2}\left(3 z^{2}-r^{2}\right) \exp \left(-r / r_{2}\right) \\
0 \\
{\left[-\left(3^{1 / 2} / 2\right) c_{2}\left(x^{2}-y^{2}\right)+i c_{3} x y\right] \exp \left(-r / r_{2}\right)}
\end{array}\right) \\
\left(\begin{array}{l}
\varphi_{13} \\
\varphi_{23} \\
\varphi_{33} \\
\varphi_{43}
\end{array}\right) & =\left(\begin{array}{c}
{\left[-\left(3^{1 / 2} / 2\right) c_{2}\left(x^{2}-y^{2}\right)-i c_{3} x y\right] \exp \left(-r / r_{2}\right)} \\
0 \\
c_{1} \exp \left(-r / r_{1}\right)-\frac{1}{2} c_{2}\left(3 z^{2}-r^{2}\right) \exp \left(-r / r_{2}\right) \\
-i c_{3} z(x+i y) \exp \left(-r / r_{2}\right) \\
0
\end{array}\right) \\
\left(\begin{array}{c}
\varphi_{14} \\
\varphi_{24} \\
\varphi_{34} \\
\varphi_{44}
\end{array}\right)= & \left(\begin{array}{c}
{\left[-\left(3^{1 / 2} / 2\right) c_{2}\left(x^{2}-y^{2}\right)-i c_{3} x y\right] \exp \left(-r / r_{2}\right)} \\
i c_{3} z(x-i y) \exp \left(-r / r_{2}\right) \\
c_{1} \exp \left(-r / r_{1}\right)+\frac{1}{2} c_{2}\left(3 z^{2}-r^{2}\right) \exp \left(-r / r_{2}\right)
\end{array}\right)
\end{aligned}
$$

where $c_{1}, c_{2}, c_{3}, r_{1}$ and $r_{2}$ are real parameters, and $r_{1}$ and $r_{2}$ are positive. The functions $7-10$ fulfill the orthonormalization condition 6 if

$$
\pi c_{1}^{2} r_{1}^{3}+9 \pi c_{2}^{2} r_{2}^{7}+(9 / 2) \pi c_{3}^{2} r_{2}^{7}=1 \text {. }
$$

As one can see from (7)-(10), the envelope functions are composed from an s-type part (that proportional to $c_{1}$ ) 
and two d-type parts (those proportional to $c_{2}$ and $c_{3}$ ). The d-type parts are responsible for mixing different $\Phi_{j}$ in a given $\psi_{i}$.

In order to determine the parameters $c_{1}, c_{2}, c_{3}, r_{1}$ and $r_{2}$, we have to maximize the mean energy (the expectation value of the acceptor's Hamiltonian) corresponding to one of the states (5), say $\psi_{1}$. This energy is

$$
E\left[\psi_{1}\right]=\sum_{k, l=1}^{4} \sum_{\alpha, \beta=1}^{3} D_{k l}^{\alpha \beta}\left\langle\varphi_{k 1}\left|\frac{\partial^{2}}{\partial x_{\alpha} \partial x_{\beta}}\right| \varphi_{l 1}\right\rangle+\frac{e^{2}}{\varepsilon} \sum_{j=1}^{4}\left\langle\varphi_{j 1}\left|\frac{1}{r}\right| \varphi_{j 1}\right\rangle,
$$

where $\varepsilon$ is the dielectric constant of the crystal and [23]

$$
\begin{aligned}
\underline{D} & =\left(\begin{array}{cccc}
\underline{D}_{11} & \underline{D}_{12} & \underline{D}_{13} & 0 \\
\underline{D}_{12}^{*} & \underline{D}_{22} & 0 & \underline{D}_{13} \\
\underline{D}_{13}^{*} & 0 & \underline{D}_{22} & -\underline{D}_{12} \\
0 & \underline{D}_{13}^{*} & -\underline{D}_{12}^{*} & \underline{D}_{11}
\end{array}\right), \\
\underline{D}_{11} & =\left(\begin{array}{ccc}
-A-\frac{1}{2} B & 0 & 0 \\
0 & -A-\frac{1}{2} B & 0 \\
0 & 0 & -A+B
\end{array}\right), \\
\underline{D}_{22} & =\left(\begin{array}{ccc}
-A+\frac{1}{2} B & 0 & 0 \\
0 & -A+\frac{1}{2} B & 0 \\
0 & 0 & -A-B
\end{array}\right), \\
\underline{D}_{12} & =\left(\begin{array}{ccc}
0 & 0 \\
0 & -\frac{1}{2} i\left(3 B^{2}+C^{2}\right)^{1 / 2} \\
-\frac{1}{2} i\left(3 B^{2}+C^{2}\right)^{1 / 2} & -\frac{1}{2}\left(3 B^{2}+C^{2}\right)^{1 / 2} & -\frac{1}{2}\left(3 B^{2}+C^{2}\right)^{1 / 2} \\
-\left(3^{1 / 2} / 2\right) B & -\frac{1}{2} i\left(3 B^{2}+C^{2}\right)^{1 / 2} & 0 \\
-1 & \left(3^{1 / 2} / 2\right) B & 0 \\
-\frac{1}{2} i\left(3 B^{2}+C^{2}\right)^{1 / 2} & 0 & 0
\end{array}\right) .
\end{aligned}
$$

$A, B$ and $C$ are material constants [24]. Signs of the elements of $D_{12}$ and $D_{13}$ involving $C^{2}$ correspond to the case of the negative value of the constant $N$ defined in [24]. If $N>0$, the signs should be opposite. However, these signs are of no importance for the following except for the sign of $c_{3} / c_{1}$. The physical meaning of $A, B$ and $C$ can be seen from the dispersion law of electrons in the valence band ( $k$-electron wave vector) :

$$
E(\underline{k})=A k^{2} \pm\left[B^{2} k^{4}+C^{2}\left(k_{x}^{2} k_{y}^{2}+k_{y}^{2} k_{z}^{2}+k_{z}^{2} k_{x}^{2}\right)\right]^{1 / 2} .
$$

$A$ and $B$ are real but $C$ may be imaginary (i.e. $C^{2}$ may be negative). In order to have $E(\underline{k})$ real and negative for all $\underline{k} \neq 0$, it should be

$$
\begin{gathered}
C^{2} \geqslant-3 B^{2}, \\
A<-\left[B^{2}+\frac{1}{3} \max \left(0, C^{2}\right)\right]^{1 / 2} .
\end{gathered}
$$

If $C=0$, there is no warping of the valence band.

From (7) and (12)-(17) we obtain after some algebra $E\left[\psi_{1}\right]$ as a quadratic form of dimensionless quantities $a_{1}, a_{2}$ and $a_{3}$, with coefficients depending on dimensionless $\rho_{1}$ and $\rho_{2}$, where

$$
\begin{aligned}
& a_{1}=\left(\pi r_{1}^{3}\right)^{1 / 2} c_{1}, \\
& a_{2}=\left(9 \pi r_{2}^{7}\right)^{1 / 2} c_{2}, \\
& a_{3}=\left(9 \pi r_{2}^{7} / 2\right)^{1 / 2} c_{3},
\end{aligned}
$$

$$
\begin{aligned}
& \rho_{1}=\frac{e^{2}}{\varepsilon|A|} r_{1}, \\
& \rho_{2}=\frac{e^{2}}{\varepsilon|A|} r_{2} .
\end{aligned}
$$

$a_{1}^{2}, a_{2}^{2}$ and $a_{3}^{2}$ are the norms of the s-type and two dtype parts of the envelope, respectively (compare with (11)).

Dividing $E\left[\psi_{1}\right]$ by $a_{1}^{2}+a_{2}^{2}+a_{3}^{2}$ and maximizing the ratio in respect to $a_{2} / a_{1}$ and $a_{3} / a_{1}$, we obtain

$$
\begin{aligned}
E\left[\psi_{1}\right] & =\frac{e^{4}}{2 \varepsilon^{2}|A|} f\left(\rho_{1}, \rho_{2} ; \eta\right), \\
a_{2}^{2} & =\frac{2}{5+(C / B)^{2}} F\left(\rho_{1}, \rho_{2} ; \eta\right), \\
a_{3}^{2} & =\frac{3+(C / B)^{2}}{5+(C / B)^{2}} F\left(\rho_{1}, \rho_{2} ; \eta\right),
\end{aligned}
$$


where the dimensionless $f, F$ and $\eta$ are

$$
\begin{gathered}
f\left(\rho_{1}, \rho_{2} ; \eta\right)=\frac{1}{\rho_{1}}\left(1-\frac{1}{\rho_{1}}\right)+\frac{1}{\rho_{2}}\left(\frac{1}{3}-\frac{1}{\rho_{2}}\right)+ \\
+\left\{\left[\frac{1}{\rho_{1}}\left(1-\frac{1}{\rho_{1}}\right)-\frac{1}{\rho_{2}}\left(\frac{1}{3}-\frac{1}{\rho_{2}}\right)\right]^{2}+\frac{1024 \eta \rho_{1}^{3} \rho_{2}\left(\rho_{1}+5 \rho_{2}\right)^{2}}{25\left(\rho_{1}+\rho_{2}\right)^{10}}\right\}^{1 / 2}, \\
F\left(\rho_{1}, \rho_{2} ; \eta\right)=\left[1+\left(\left[\frac{1}{\rho_{2}}\left(\frac{1}{3}-\frac{1}{\rho_{2}}\right)-\frac{1}{\rho_{1}}\left(1-\frac{1}{\rho_{1}}\right)\right] \times\right.\right. \\
\quad \times \frac{5\left(\rho_{1}+\rho_{2}\right)^{5}}{32 \eta^{1 / 2} \rho_{1}^{3 / 2} \rho_{2}^{1 / 2}\left(\rho_{1}+5 \rho_{2}\right)}+\left\{1+\left[\frac{1}{\rho_{2}}\left(\frac{1}{3}-\frac{1}{\rho_{2}}\right)-\frac{1}{\rho_{1}}\left(1-\frac{1}{\rho_{1}}\right)\right]^{2}\right. \\
\left.\left.\left.\times \frac{25\left(\rho_{1}+\rho_{2}\right)^{10}}{1024 \eta \rho_{1}^{3} \rho_{2}\left(\rho_{1}+5 \rho_{2}\right)^{2}}\right\}^{1 / 2}\right)^{-2}\right]^{-1}, \\
\eta=\frac{5+(C / B)^{2}}{2(A / B)^{2}} .
\end{gathered}
$$

The sign of $a_{2} / a_{1}$ is that of $B . a_{3} / a_{1}$ is positive (negative if the constant $N$ of [24] is positive). It follows from (19) and (20) that

$$
0<\eta<\frac{5}{2} \text {. }
$$

It is worth noting that in the absence of warping (i.e. for $C=0$ ) $\eta$ is determined by the ratio of heavy and light hole effective masses

$$
\mu=m_{\mathrm{hh}} / m_{\mathrm{lh}} \text {. }
$$

Therefore, we obtain from (18) and (31)

$$
\eta=\frac{5}{2}\left(\frac{\mu-1}{\mu+1}\right)^{2}
$$

From (27) and (28) we then obtain

$$
a_{3}^{2} / a_{2}^{2}=\frac{3}{2} \text {. }
$$

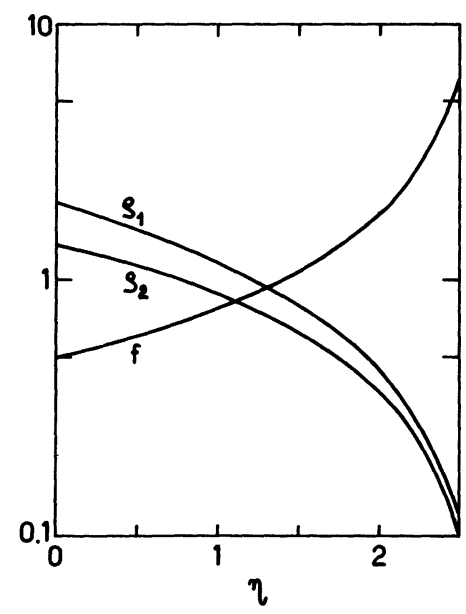

Fig. 1. - The maximal value of the function $f$ defined by (29) and the corresponding values of the variables $\rho_{1}$ and $\rho_{2}$, for different values of the parameter $\eta$.
The inequality 20 imposes some limitations on the value of $(C / B)^{2}$, at a given $\eta$, namely,

$$
\begin{aligned}
\max (-3,2 \eta-5)<(C / B)^{2} \\
< \\
\left.<\max \left[0,\left(\frac{2}{3} \eta-1\right) / 2\left(\frac{5}{2}-\eta\right)\right]\right\}^{-1} .
\end{aligned}
$$

In order to maximize $E\left[\psi_{1}\right]$ given by (26), we have to maximize $f\left(\rho_{1}, \rho_{2} ; \eta\right)$ for a given $\eta$. This was done numerically for all values of $\eta$ in the range (32). The optimal values of $\rho_{1}$ and $\rho_{2}$ and the maximal value of $f$ (all being now functions of $\eta$ ) are shown in figure 1. Inserting the optimal values of $\rho_{1}$ and $\rho_{2}$ in (30) we obtain the function $F(\eta)$ shown in figure 2 .

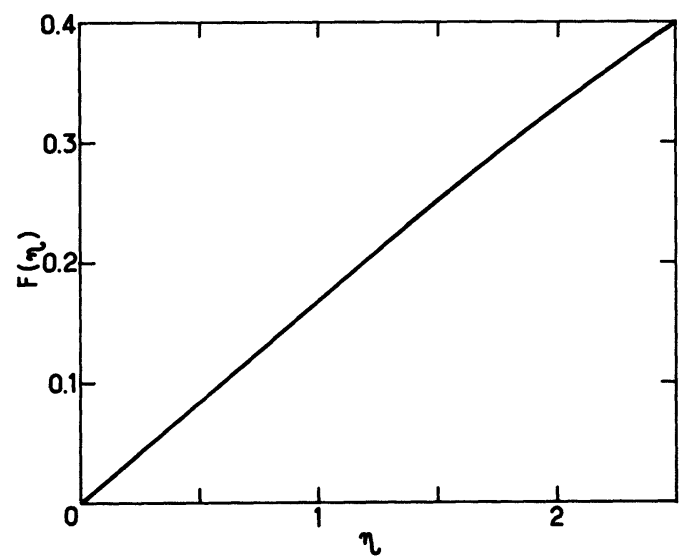

Fig. 2. - The function $F$ defined by (30) for $\rho_{1}$ and $\rho_{2}$ maximizing the function $f$, for different values of the parameter $\boldsymbol{\eta}$.

Substituting $f=f(\eta)$ and $F=F(\eta)$ in the formulas 26-28 we obtain the acceptor binding energy and the norms of the two d-type parts of the envelope. 


\section{Exchange splitting.}

We will now take into account the exchange interaction of the band electrons with the magnetic ions (e.g., with the $3 \mathrm{~d}^{5}$-electrons of $\mathrm{Mn}^{2+}$ ions in $\left.\mathrm{Cd}_{1-x} \mathrm{Mn}_{x} \mathrm{Te}\right)$. This interaction is of Heisenberg form

$$
H_{\text {exch }}=\underline{s} \cdot \sum_{\underline{R}_{i}} \underline{S}_{i} J\left(\underline{r}-\underline{R}_{i}\right),
$$

where $\underline{s}$ and $\underline{S}_{i}$ are the spin operators (in units of $\hbar$ ) of the band electron and of a magnetic ion, respectively, and the summation is over all lattice sites occupied by magnetic ions. $J$ is a short-range function of distance.

Using the molecular-field and virtual-crystal approximations we can write (37) in the form (see, e.g., [8-10])

$$
H_{\text {exch }}=x \underline{s} \cdot\langle\underline{S}\rangle_{\text {av }} \sum_{\underline{R}} J(\underline{r}-\underline{R}),
$$

which has the periodicity of the crystal lattice, as the summation is over all metal lattice sites. $x$ is the molar fraction of the magnetic component and $\langle\underline{S}\rangle_{\mathrm{av}}$ is the spin of a magnetic ion averaged thermally and over all magnetic ions. In the absence of an external magnetic field, $\langle\underline{S}\rangle_{\mathrm{av}}$ vanishes. In the presence of a magnetic field it increases with decreasing temperature. In the following we will assume $\langle\underline{S}\rangle_{\text {av }} \| z$, i.e. the external magnetic field parallel to the $z$-direction.

The exchange Hamiltonian (38) written in the basis (1)-(4) has the form

$$
H_{\text {exch }}=\left(\begin{array}{cccc}
3 B_{\text {exch }} & 0 & 0 & 0 \\
0 & B_{\text {exch }} & 0 & 0 \\
0 & 0 & -B_{\text {exch }} & 0 \\
0 & 0 & 0 & -3 B_{\text {exch }}
\end{array}\right),
$$

where

$$
\begin{aligned}
B_{\mathrm{exch}} & =\frac{1}{6} x N_{0} \beta\left\langle S_{z}\right\rangle_{\mathrm{av}}, \\
\beta & =\langle Z|J| Z\rangle,
\end{aligned}
$$

and $N_{0}$ is the number of unit cells in unit volume. Usually $N_{0} \beta \sim 1 \mathrm{eV}$ and $2 B_{\text {exch }} \sim-10 \mathrm{meV}$ (at helium temperatures and few tesła). $B_{\text {exch }}$ may also include the Luttinger parameter $\kappa$ [25].

In the wide-gap semimagnetic semiconductors, because of the high effective masses of holes, the acceptor binding energy (in the absence of exchange) is much higher than the exchange splitting $6\left|B_{\text {exch }}\right|$ of $\Gamma_{8}$ level. This exchange splitting is, in turn, much higher than the Landau splitting. Therefore, it is justified to treat the exchange interaction as a perturbation in the acceptor problem, neglecting the direct effect of the magnetic field on orbital motion (i.e. on the envelope functions).
It follows from (39) that the exchange Hamiltonian written in the basis of the acceptor ground state functions 5 has the form

$$
\begin{aligned}
\left(H_{\mathrm{exch}}\right)_{i i^{\prime}} & =\left(3\left\langle\varphi_{1 i} \mid \varphi_{1 i^{\prime}}\right\rangle+\left\langle\varphi_{2 i} \mid \varphi_{2 i^{\prime}}\right\rangle\right. \\
- & \left.\left\langle\varphi_{3 i} \mid \varphi_{3 i^{\prime}}\right\rangle-3\left\langle\varphi_{4 i} \mid \varphi_{4 i^{\prime}}\right\rangle\right) B_{\mathrm{exch}}
\end{aligned}
$$

Inserting here the functions $7-10$ we obtain after some algebra

$$
\begin{gathered}
H_{\text {exch }}= \\
\left(\begin{array}{cccc}
\sigma_{1} B_{\text {exch }} & 0 & 0 & 0 \\
0 & \sigma_{2} B_{\text {exch }} & 0 & 0 \\
0 & 0 & -\sigma_{2} B_{\text {exch }} & 0 \\
0 & 0 & 0 & -\sigma_{1} B_{\text {exch }}
\end{array}\right),
\end{gathered}
$$

where (using (22) and (23)) we have

$$
\begin{aligned}
& \sigma_{1}=3-2 a_{2}^{2}-\frac{8}{3} a_{3}^{2}, \\
& \sigma_{2}=1-2 a_{2}^{2} .
\end{aligned}
$$

It follows from (43) that the functions 5, 7-10 are eigenfunctions also in the presence of exchange. However, the four-fold degenerate ground state of the acceptor is split by exchange. Comparing (39) with (43)-(45) one can see that the acceptor splitting is smaller than the splitting of the free-carrier $\Gamma_{8}$ state, and that the split levels are not equidistant. This quenching of the acceptor's splitting results from the fact that the acceptor wave function is composed of different functions of the $\Gamma_{8}$ level (with d-type envelopes).

The dependence (44), (45), of $\sigma_{1}$ and $\sigma_{2}$ on $a_{2}^{2}$ and $a_{3}^{2}$ is shown in the form of a map in figure 3 .

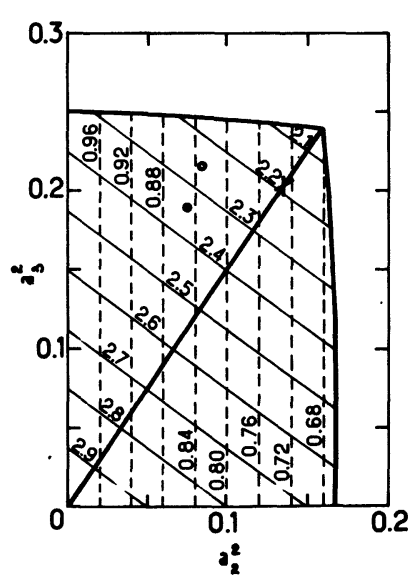

Fig. 3. - Acceptor splitting as a function of the norms of the d-type envelope components. Bold lines : the boundaries (46) and (47) of the region of points representing all possible materials, and the nowarping line (35). Continuous lines correspond to the indicated values of $\sigma_{1}$, and broken lines to the indicated values of $\sigma_{2}$. Full circle : Ge, empty circle : CdTe, cross : $\mathrm{Hg}_{0.87} \mathrm{Mn}_{0.13} \mathrm{Te}$. 
The line (35) is also drawn. The point corresponding to a given material will be situated on this line if there is no warping. The region of points representing all possible materials is limited at the top by the curve

$$
a_{2}^{2}=\left(1-\frac{3}{2 \eta}\right) F(\eta), \quad\left(\frac{3}{2} \leqslant \eta<\frac{5}{2}\right)
$$

and on the right-hand side by the curve

$$
\begin{aligned}
& a_{2}^{2}=\frac{1}{\eta} F(\eta), \\
& a_{3}^{2}=\left(1-\frac{1}{\eta}\right) F(\eta) .
\end{aligned}
$$

The limitations follow from (27), (28) and (36).

Substituting (27) and (28) into (44) and (45), we obtain $\sigma_{1}$ and $\sigma_{2}$ as the functions of the material parameters $\eta$ and $(C / B)^{2}$ :

$$
\begin{aligned}
& \sigma_{1}=3-\frac{12+(8 / 3)(C / B)^{2}}{5+(C / B)^{2}} F(\eta) \\
& \sigma_{2}=1-\frac{4}{5+(C / B)^{2}} F(\eta)
\end{aligned}
$$

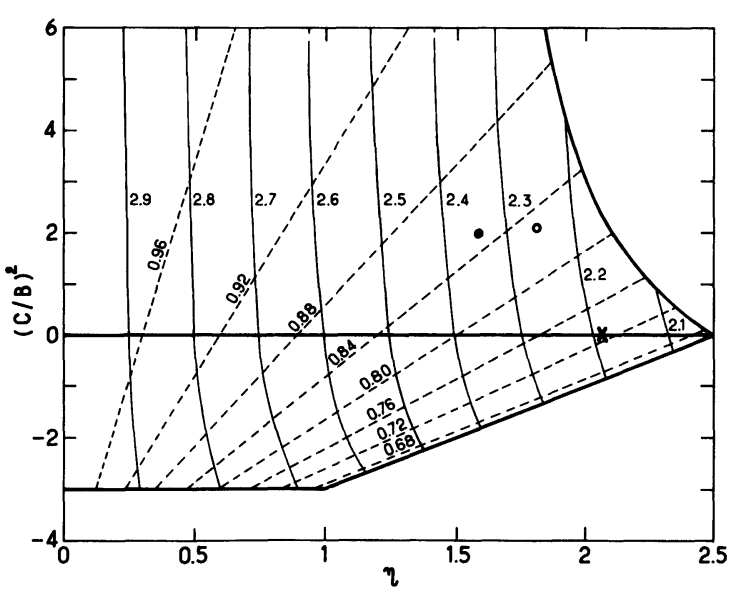

Fig. 4. - Acceptor splitting as a function of the material parameters. Bold lines : the boundaries (36) of the region of points representing all possible materials, and the nowarping line $C=0$. Continuous lines correspond to the indicated values of $\sigma_{1}$, and broken lines to the indicated values of $\sigma_{2}$. Full circle : Ge, open circle : CdTe, cross : $\mathrm{Hg}_{0.87} \mathrm{Mn}_{0.13} \mathrm{Te}$.

These functions are shown in the form of a map in figure 4. The region of points representing all possible materials is limited by (36).

Some numerical examples (not necessarily semi- magnetic or wide-gap) are included in figures 3 and 4. Those correspond to the following materials :

$$
\begin{aligned}
& \mathrm{Ge}:|A|=13.2 \hbar^{2} / 2 m_{0}, \\
&|B|=8.9 \hbar^{2} / 2 m_{0}, \\
&|C|=12.5 \hbar^{2} / 2 m_{0}, \\
& \mathrm{CdTe}:|A|=5.29 \hbar^{2} / 2 m_{0}, \\
&|B|=3.78 \hbar^{2} / 2 m_{0}, \\
&|C|=5.45 \hbar^{2} / 2 m_{0}, \\
& \mathrm{Hg}_{0.87} \mathrm{Mn}_{0.13} \mathrm{Te}:|A|=27.46 \hbar^{2} / 2 m_{0},|B|=24.96 \hbar^{2} / 2 m_{0}, \\
&|C|=0,
\end{aligned}
$$

where $m_{0}$ is the free-electron mass.

\section{Acceptor to conduction Landau states transitions.}

We will calculate the matrix elements of the optical transitions of the electron from the acceptor ground state to the $\Gamma_{6}$-conduction-band Landau levels. As the ground state wave functions 5,7-10 are not mixed by the exchange Hamiltonian $H_{\text {exch }}$, the results are independent of exchange, except for the energy distances between the initial and final states which are influenced by both the acceptor and conduction band exchange splitting.

The Landau quantization of the conduction band influences the absorption spectrum qualitatively making it discrete. Thus, it seems justified to take it into account, while neglecting the quantitative corrections to the acceptor states due to the Lorentz force.

We use the Landau gauge for the vector potential, i.e.

$$
\underline{A}=(0, H x, 0),
$$

where $(0,0, H)$ is the magnetic field intensity. The conduction-band orthonormalized Landau states are

$$
\begin{aligned}
& |n \uparrow\rangle=|S \uparrow\rangle f_{n}(\underline{r}), \\
& |n \downarrow\rangle=i|S \downarrow\rangle f_{n}(\underline{r}),
\end{aligned}
$$

where the position-dependent function $S$ transforms under the operations of the $T_{\mathrm{d}}$ point group to an s-type function and has the average square modulus equal to 1 . The envelope function $f_{n}$ is

$$
f_{n}(r)=\left(L_{y} L_{z}\right)^{-1 / 2} \exp \left[i\left(k_{y} y+k_{z} z\right)\right] g_{n}\left(\frac{x}{\lambda}+\lambda k_{y}\right),
$$

where $\lambda=(\hbar c / e H)^{1 / 2}$ is the magnetic length,

$$
g_{n}(u)=\left(\pi^{1 / 2} \lambda 2^{n} n !\right)^{-1 / 2} \exp \left(-\frac{1}{2} u^{2}\right) H_{n}(u),
$$

and $H_{n}(u)$ is the Hermite polynomial

$$
H_{n}(u)=(-1)^{n} \exp \left(u^{2}\right) \frac{\mathrm{d}^{n}}{\mathrm{~d} u^{n}} \exp \left(-u^{2}\right) .
$$


The function $f_{n}(r)$ is normalized in the periodicity box of the dimensions $L_{y}, L_{z}$ in the directions $y, z$, respectively. $k_{y}$ and $k_{z}$ are quantized : $k_{\alpha}=\left(2 \cdot \pi / L_{\alpha}\right) K_{\alpha}(\alpha=$ $y, z)$ where $K_{\alpha}$ are integers.

The matrix element of the optical transition of the electron from the acceptor ground state $i$ to the conduction-band Landau level is of the form

$$
\langle n \uparrow \downarrow|\underline{E} \cdot \underline{\pi}| i\rangle
$$

where $\underline{E}$ is the complex amplitude of the uniform electric field of radiation

$$
\operatorname{Re}[\underline{E} \exp (-i \omega t)] \text {, }
$$

and (p-momentum operator)

$$
\underline{\pi}=\underline{p}+\frac{e}{c} \underline{A}
$$

Inserting (5), (51), (52), and (58) into (56), we obtain

$$
\begin{aligned}
&\langle n \uparrow|\underline{E} \cdot \underline{\pi}| i\rangle=\sum_{j=1}^{4}\left(E_{z}\left\langle S \uparrow\left|p_{z}\right| \Phi_{j}\right\rangle\left\langle f_{n} \mid \varphi_{j i}\right\rangle+\right. \\
&+\left.E^{+}\left\langle S \uparrow\left|p^{-}\right| \Phi_{j}\right\rangle\left\langle f_{n} \mid \varphi_{j i}\right\rangle+E^{-}\left\langle S \uparrow\left|p^{+}\right| \Phi_{j}\right\rangle\left\langle f_{n} \mid \varphi_{j i}\right\rangle\right), \\
&\langle n \downarrow|\underline{E} \cdot \underline{\pi}| i\rangle=-i \sum_{j=1}^{4}\left(E_{z}\left\langle S \downarrow\left|p_{z}\right| \Phi_{j}\right\rangle\left\langle f_{n} \mid \varphi_{j i}\right\rangle+\right. \\
&\left.+E^{+}\left\langle S \downarrow\left|p^{-}\right| \Phi_{j}\right\rangle\left\langle f_{n} \mid \varphi_{j i}\right\rangle+E^{-}\left\langle S \downarrow\left|p^{+}\right| \Phi_{j}\right\rangle\left\langle f_{n} \mid \varphi_{j i}\right\rangle\right),
\end{aligned}
$$

where

$$
\begin{aligned}
E^{ \pm} & =2^{-1 / 2}\left(E_{x} \pm i E_{y}\right), \\
p^{ \pm} & =2^{-1 / 2}\left(p_{x} \pm i p_{y}\right)
\end{aligned}
$$

Integrals involving $S$ are over a unit volume, and those with envelopes are over the whole space. The only six non-vanishing matrix elements of $p_{z}, p^{ \pm}$are (see (1)-(4))

$$
\begin{aligned}
& \left\langle S \uparrow\left|p_{z}\right| \Phi_{2}\right\rangle=-i\left\langle S \downarrow\left|p_{z}\right| \Phi_{3}\right\rangle=-(2 / 3)^{1 / 2} i P, \\
& \left\langle S \uparrow\left|p^{-}\right| \Phi_{1}\right\rangle=-i\left\langle S \downarrow\left|p^{+}\right| \Phi_{4}\right\rangle=P, \\
& \left\langle S \uparrow\left|p^{+}\right| \Phi_{3}\right\rangle=-i\left\langle S \downarrow\left|p^{-}\right| \Phi_{2}\right\rangle=3^{-1 / 2} P,
\end{aligned}
$$

where

$$
P=\left\langle S\left|p_{z}\right| Z\right\rangle
$$

From (53) and (54) we obtain

$$
\begin{aligned}
\left\langle f_{n} \mid \varphi_{j i}\right\rangle=\left(\pi^{1 / 2} \lambda 2^{n} n ! L_{y} L_{z}\right)^{-1 / 2} & \exp \left[-\frac{1}{2}\left(\lambda k_{y}\right)^{2}\right] \times \\
\times & \left\langle\exp \left[-\frac{1}{2}\left(\frac{x}{\lambda}\right)^{2}-k_{y}(x-i y)\right] \exp \left(i k_{z} z\right) H_{n}\left(\frac{x}{\lambda}+\lambda k_{y}\right) \mid \varphi_{j i}(r)\right\rangle .
\end{aligned}
$$

Due to the presence of $\varphi_{j i}$, the contributions to the integral are given only by $|\underline{r}| \sim r_{1}, r_{2}$ (see (7)-(10)). On the other hand, due to the pre-bracket factor, we are not interested in $k_{y} \gg 1 / \lambda$. Thus, if

$$
\lambda \gg r_{1}, r_{2},
$$

we can put in (67)

$$
\exp \left[-\frac{1}{2}\left(\frac{x}{\lambda}\right)^{2}-k_{y}(x-i y)\right] \cong 1
$$

We can also neglect $x / \lambda$ in the argument of $H_{n}$, obtaining

$$
\left\langle f_{n} \mid \varphi_{j i}\right\rangle=\left(\pi^{1 / 2} \lambda 2^{n} n ! L_{y} L_{z}\right)^{-1 / 2} \exp \left[-\frac{1}{2}\left(\lambda k_{y}\right)^{2}\right] H_{n}\left(\lambda k_{y}\right) \int \exp \left(-i k_{z} z\right) \varphi_{j i}(x) \mathrm{d}^{3} r
$$


The assumption (68) was already used by us implicitly when neglecting the influence of the magnetic field on the envelope functions of the acceptor. For wide-gap semiconductors it is fulfilled up to the magnetic field of the order of $10 \mathrm{~T}$.

It follows from (7)-(10) that

$$
\begin{aligned}
& \int \exp \left(-i k_{z} z\right) \varphi_{j i} \underline{(r)} \mathrm{d}^{3} r=0 \quad \text { for } \quad j \neq i, \\
& \int \exp \left(-i k_{z} z\right) \varphi_{i i}(\underline{r}) \mathrm{d}^{3} r=8 \pi G_{ \pm}\left(k_{z}^{2}\right),
\end{aligned}
$$

where " + " in (72) corresponds to $i=2,3$, and " - " corresponds to $i=1,4$, and where we have denoted

$$
G_{ \pm}\left(k_{z}^{2}\right)=r_{1}^{3} c_{1}\left[1+\left(k_{z} r_{1}\right)^{2}\right]^{-2} \pm 24 r_{2}^{5} c_{2}\left(k_{z} r_{2}\right)^{2}\left[1+\left(k_{z} r_{2}\right)^{2}\right]^{-4} \text {. }
$$

What appears in the formula for the absorption coefficient is the square modulus of the matrix element summed over $k_{y}$. Inserting (63)-(65) and (70)-(72) into (59) and (60) we obtain finally

$$
\begin{aligned}
& \sum_{k_{y}}|\langle n \uparrow|\underline{E} \cdot \underline{\pi}| 4\rangle|^{2}=\sum_{k_{y}}|\langle n \downarrow|\underline{E} \cdot \underline{\pi}| 1\rangle|^{2}=0, \\
& \sum_{k_{y}}|\langle n \uparrow|\underline{E} \cdot \underline{\pi}| 2\rangle|^{2}=\sum_{k_{y}}|\langle n \downarrow|\underline{E} \cdot \underline{\pi}| 3\rangle|^{2}=\frac{32 \pi}{\lambda^{2} L_{z}}|P|^{2}\left|E_{z}\right|^{2} \frac{2}{3} G_{+}^{2}\left(k_{z}^{2}\right), \\
& \sum_{k_{y}}|\langle n \uparrow|\underline{E} \cdot \underline{\pi}| 1\rangle|^{2}=\frac{32 \pi}{\lambda^{2} L_{z}}|P|^{2}\left|E^{+}\right|^{2} G_{-}^{2}\left(k_{z}^{2}\right), \\
& \sum_{k_{y}}|\langle n \downarrow|\underline{E} \cdot \underline{\pi}| 2\rangle|^{2}=\frac{32 \pi}{\lambda^{2} L_{z}}|P|^{2}\left|E^{+}\right|^{2} \frac{1}{3} G_{+}^{2}\left(k_{z}^{2}\right), \\
& \sum_{k_{y}}|\langle n \uparrow|\underline{E} \cdot \underline{\pi}| 3\rangle|^{2}=\frac{32 \pi}{\lambda^{2} L_{z}}|P|^{2}\left|E^{-}\right|^{2} \frac{1}{3} G_{+}^{2}\left(k_{z}^{2}\right), \\
& \sum_{k_{y}}|\langle n \downarrow|\underline{E} \cdot \underline{\pi}| 4\rangle|^{2}=\frac{32 \pi}{\lambda^{2} L_{z}}|P|^{2}\left|E^{-}\right|^{2} G_{-}^{2}\left(k_{z}^{2}\right) .
\end{aligned}
$$

As $c_{1}$ and $c_{2}$ are usually of opposite $\operatorname{sign}$ ( $B$ is usually negative), $G_{-}^{2}>G_{+}^{2}$. Thus, the strongest transitions are $1 \rightarrow n \uparrow\left(E^{+}\right)$and $4 \rightarrow n \downarrow\left(E^{-}\right)$.

\section{Acknowledgments.}

The research of one of the authors (J. M.) was supported in part by Institute of Physics, Polish Academy of Sciences.

\section{References}

[1] Galazka, R. R., Physics of Semiconductors, ed. B. L. H. Wilson (The Institute of Physics, Bristol) 1979, Conf. Ser. 43, p. 133.

[2] Galazka, R. R. and Kossut, J., Proc. Int. School Narrow-Gap Semicond., Nîmes, France 1979, p. 245.

[3] Gaj, J. A., Proc. 15th Int. Conf. Phys. Semicond. Kyoto (1980), ed. S. Tanaka and Y. Toyozawa, J. Phys. Soc. Japan 49 (1980) Suppl. A, p. 797.

[4] Bastard, G., Gaj, J. A., Planel, R. and Rigaux, C., J. Physique Colloq. 41 (1980) C5-247.

[5] Mycielski, J., Recent Developments in Condensed Matter Physics, Vol. 1, ed. J. T. Devreese (Plenum Press, New York) 1981, p. 725.
[6] Dietl, T., Physics in High Magnetic Fields, ed. M. Miura, Springer Ser. Solid State Sciences, Vol. 24 (Springer, Berlin) 1981, p. 344.

[7] Galazka, R. R., Physics of Narrow Gap Semiconductors, Proc. 4th Int. Conf. Linz 1981, ed. E. Gornik, H. Heinrich and L. Palmetshofer (Springer, Berlin) 1982, p. 294

[8] Mycielski, J., Proc. Int. Conf. The Application of High Magnetic Fields in Semiconductor Physics, Grenoble (1982), in press.

[9] Bastard, G., RigauX, C., Guldner, Y., Mycielski, J. and MyCielski, A., J. Physique 39 (1978) 87.

[10] JACZYNSKI, M., Kossut, J. and GalazKa, R. R., Phys. Status Solidi (b) 88 (1978) 73. 
[11] Gaj, J. A., Ginter, J. and Galazka, R. R., Phys. Status Solidi (b) 89 (1978) 655.

[12] Delves, R. T., Proc. Phys. Soc. 87 (1966) 809.

[13] Morrissy, J. H., Ph. D. Thesis, Oxford University (1971).

[14] Mycielski, A. and Mycielski, J., Proc. 15th Int. Conf. Phys. Semicond. Kyoto (1980), ed. S. Tanaka and Y. Toyozawa, J. Phys. Soc. Japan 49 (1980) Suppl. A, p. 807.

[15] Wojtowicz, T. and Mycielski, A., Proc. 16th Int. Conf. Phys. Semicond. Montpellier (1982), in press.

[16] JARoszyŃski, J., Dietl, T., SAWICki, M. and JANIK, E., Proc. 16th Int. Conf. Phys. Semicond. Montpellier (1982), in press.

[17] Schechter, D., J. Phys. Chem. Solids 23 (1962) 237.
[18] Gawron, T. R. and Trylski, J., Physics of Narrow Gap Semiconductors, Proc. 4th Int. Conf. Linz 1981, ed. E. Gornik, H. Heinrich and L. Palmetshofer (Springer, Berlin) 1982, p. 312.

[19] Baldereschi, A. and Lipari, N. O., Phys. Rev. B 8 (1973) 2697.

[20] Lipari, N. O. and Altarelli, M., Solid State Commun. 33 (1980) 47.

[21] Planel, R., Proc. Int. Conf. The Application of High Magnetic Fields in Semiconductor Physics, Grenoble (1982), in press.

[22] Kaczmarex, E., Acta Phys. Polonica 30 (1966) 267.

[23] Luttinger, J. M. and KoHN, W., Phys. Rev. 97 (1955) 869.

[24] Dresselhaus, G., KiP, A. F. and Kittel, C., Phys. Rev. 98 (1955) 368.

[25] Luttinger, J. M., Phys. Rev. 102 (1956) 1030. 